\title{
BOUNDS FOR THE GEOMETRIC-ARITHMETIC INDEX OF A GRAPH
}

\author{
JOSÉ M. SIGARRETA
}

Received 13 November, 2014

\begin{abstract}
The first geometric-arithmetic index $G A_{1}(G)$, which was introduced by D. Vukičević and $\mathrm{B}$. Furtula recently, is a graph-based molecular structure descriptor. The aim of this paper is to obtain new inequalities involving the geometric-arithmetic index $G A_{1}$ and other well known topological indices. In particular, we relate the geometric-arithmetic index of a graph $G$ with its first Zagreb index $M_{1}(G)$, its second Zagreb index $M_{2}(G)$, its modified Zagreb index $Z_{-1}(G)$ and its Randić index $R(G)$.
\end{abstract}

2010 Mathematics Subject Classification: 05C07; 92E10

Keywords: graph invariant, inequalities, topological index, geometric-arithmetic index

\section{INTRODUCTION}

The study of topological indices is a subject of increasing interest, both in pure and applied mathematics. Topological indices are interesting since they capture some of the properties of a molecule (or a graph) in a single number(see, e.g., [17, 22]). Hundreds of topological indices have been introduced and studied, starting with the seminal work by Wiener [19] in which he used the sum of all shortest-path distances of a (molecular) graph for modeling physical properties of alkanes. Probably, the Randić index $R(G)$ is the best know topological index (see, e.g., [5,9,10,14,15] and the references cited therein). The Randić index $R(G)$ is defined in [14] as

$$
R=R(G)=\sum_{u v \in E(G)} \frac{1}{\sqrt{d_{u} d_{v}}}
$$

During many years, scientists were trying to improve the predictive power of the Randić index. This led to the introduction of a large number of new topological

Supported in part by a grant from Ministerio de Economía y Competititvidad (MTM 2013-46374-P), Spain, and by a grant from CONACYT (2015) (UAGro), Mexico. 
descriptors resembling the original Randić index. The first geometric-arithmetic index $G A_{1}(G)$, defined in [18] as

$$
G A_{1}=G A_{1}(G)=\sum_{u v \in E(G)} \frac{\sqrt{d_{u} d_{v}}}{\frac{1}{2}\left(d_{u}+d_{v}\right)},
$$

where $u v$ denotes the edge of the graph $G$ connecting the vertices $u$ and $v$, and $d_{u}$ is the degree of the vertex $u$, is one of the successors of the Randic index. Although $G A_{1}$ was introduced just five years ago, there are many papers dealing with this index. There are other geometric-arithmetic indices, like $Z_{p, q}\left(Z_{0,1}=G A_{1}\right)$, but the results in [2] show empirically that the $G A_{1}$ index gathers the same information on observed molecules as other $Z_{p, q}$ indices.

Topological indices based on end-vertex degrees of edges have been used over thirty years. Among them, several indices are recognized to be useful tools in chemical researches. The graph based molecular descriptors called Zagreb indices were introduced by Gutman and Trinajstić. Since then, several results concerning Zagreb indices have been communicated in the chemical and mathematical literature (see $[1,4,6,13,21]$ and the references cited therein). The variable Zagreb index is defined in $[11]$ as

$$
Z_{\alpha}=Z_{\alpha}(G)=\sum_{u v \in E(G)}\left(d_{u} d_{v}\right)^{\alpha},
$$

and was used in the structure-boiling point modeling of benzenoid hydrocarbons. The obtained model is practically identical to the model based on the variable vertexconnectivity index and this is due to close relationship between the formulas for the two indices.

The reason for introducing a new index is to gain prediction of some property of molecules somewhat better than obtained by already presented indices. Therefore, a test study of predictive power of a new index must be done. As a standard for testing new topological descriptors, the properties of octanes are commonly used. We can find 16 physico-chemical properties of octanes at www.moleculardescriptors.eu. The $G A_{1}$ index gives better correlation coefficients than Randić index for these properties, but the differences between them are not significant. However, the predicting ability of the $G A_{1}$ index compared with Randić index is reasonably better (see [2, Table 1]).

We begin by stating some notation and terminology. Let $G=(V, E)=$ $(V(G), E(G))$ denotes a (non-oriented) finite simple connected graph of order $n=$ $|V(G)|$ and size $m=|E(G)|$ with $E(G) \neq \varnothing$. We denote two adjacent vertices $u$ and $v$ by $u \sim v$. For a vertex $u \in V$ we denote $N(v)=\{u \in V: u \sim v\}$. The degree of a vertex $v \in V$ will be denoted by $d_{v}=|N(v)|$. We denote by $\delta$ and $\Delta$ the minimum and maximum degree of the graph, respectively. We use the classical notation $u v$ for the edge of a graph joining the vertices $u$ and $v$. Note that the connectivity of $G$ is not an important restriction, since if $G$ has connected components $G_{1}, \ldots, G_{r}$, then $G A_{1}(G)=G A_{1}\left(G_{1}\right)+\cdots+G A_{1}\left(G_{r}\right)$. 


\section{Results}

We start with the following basic results. In [18] (see also [2]) the following inequalities appear :

In [2] we find the bounds

$$
\begin{gathered}
G A_{1}(G) \geq \frac{2(n-1)^{3 / 2}}{n} . \\
G A_{1}(G) \geq \frac{2 m}{n} .
\end{gathered}
$$

$$
\frac{2 m \sqrt{\Delta \delta}}{\Delta+\delta} \leq G A_{1}(G) \leq m .
$$

In [16] the authors obtain a lower bound of $G A_{1}(G)$, depending just on $n$ and $m$, for every graph $G$.

$$
G A_{1}(G) \geq \frac{2 m \sqrt{n-1}}{n} .
$$

Moreover, [16] provides a lower bound of $G A_{1}(G)$ for every graph $G$ with $\delta \geq k$, for any fixed $k \geq 2$.

(1) If $n \leq 10$, then $G A_{1}(G) \geq \frac{n k}{2}$.

(2) If $n \geq 11$, then $G A_{1}(G) \geq \min \left\{\frac{n k}{2}, \frac{(k+1) \sqrt{k}(n-1)^{3 / 2}}{n-1+k}\right\}$.

We will denote by $M_{1}(G)$ and $M_{2}(G)$ the first and the second Zagreb indices of the graph $G$, respectively, defined in [7] as

$$
M_{1}(G)=\sum_{u \in V(G)} d_{u}^{2}, \quad M_{2}(G)=\sum_{u v \in E(G)} d_{u} d_{v} .
$$

These indices have attracted growing interest, see e.g., [20] and [7].

In [3] (see also [2]) we find the bound:

$$
G A_{1}(G) \leq \frac{\sqrt{m M_{2}(G)}}{\delta} .
$$

A similar lower bound is obtained in [16]:

$$
G A_{1}(G) \geq \frac{2}{\Delta+\delta} \sqrt{\frac{\delta m M_{2}(G)}{\Delta}} .
$$

Theorem 1 below improves these lower bounds. In order to prove it, we need the following lemmas (see [16] and [8]).

Lemma 1. Let $g$ be the function $g(x, y)=\frac{2 \sqrt{x y}}{x+y}$ with $0<a \leq x, y \leq b$. Then

$$
\frac{2 \sqrt{a b}}{a+b} \leq g(x, y) \leq 1 .
$$


The equality in the lower bound is attained if and only if either $x=a$ and $y=b$, or $x=b$ and $y=a$, and the equality in the upper bound is attained if and only if $x=y$. Besides, $g(x, y)=g\left(x^{\prime}, y^{\prime}\right)$ if and only if $x / y$ is equal to either $x^{\prime} / y^{\prime}$ or $y^{\prime} / x^{\prime}$.

Proof. Let $f$ be the function $f(t)=\frac{2 t}{1+t^{2}}$ on the interval $[0, \infty)$. Then $f$ strictly increases in [0,1], strictly decreases in $[1, \infty), f(t)=1$ if and only if $t=1$ and $f(t)=f\left(t_{0}\right)$ if and only if either $t=t_{0}$ or $t=t_{0}^{-1}$. The statements follow from $f^{\prime}(t)=\frac{2\left(1-t^{2}\right)}{\left(1+t^{2}\right)^{2}}$. Now, taking $g(x, y)=f(t)$ with $t=\sqrt{\frac{x}{y}}$, and $\sqrt{\frac{a}{b}} \leq t \leq \sqrt{\frac{b}{a}}$, the result follows.

The following result provides a converse of Cauchy-Schwarz inequality.

Lemma 2. If $0<n_{1} \leq a_{j} \leq N_{1}$ and $0<n_{2} \leq b_{j} \leq N_{2}$ for $1 \leq j \leq k$, then

$$
\left(\sum_{j=1}^{k} a_{j}^{2}\right)^{1 / 2}\left(\sum_{j=1}^{k} b_{j}^{2}\right)^{1 / 2} \leq \frac{1}{2}\left(\sqrt{\frac{N_{1} N_{2}}{n_{1} n_{2}}}+\sqrt{\frac{n_{1} n_{2}}{N_{1} N_{2}}}\right)\left(\sum_{j=1}^{k} a_{j} b_{j}\right) .
$$

Theorem 1. We have for any graph $G$

$$
\frac{2 \sqrt{2 \sqrt{\Delta \delta}(\Delta+\delta) m M_{2}(G)}}{\Delta(\sqrt{\Delta}+\sqrt{\delta})^{2}} \leq G A_{1}(G) \leq \frac{M_{2}(G)}{\delta^{2}},
$$

and the equality in each inequality is attained if and only if $G$ is a regular graph.

Proof. By Lemma 1, taking $a=\delta$ and $b=\Delta$, we have

$$
\frac{2 \sqrt{\Delta \delta}}{\Delta+\delta} \leq \frac{\sqrt{d_{u} d_{v}}}{\frac{1}{2}\left(d_{u}+d_{v}\right)} \leq 1
$$

Lemma 2 gives

$$
\begin{aligned}
G A_{1}(G) & =\sum_{u v \in E(G)} \frac{\sqrt{d_{u} d_{v}}}{\frac{1}{2}\left(d_{u}+d_{v}\right)} \geq \frac{\left(\sum_{\left.u v \in E(G) \frac{4 d_{u} d_{v}}{\left(d_{u}+d_{v}\right)^{2}}\right)^{1 / 2}\left(\sum_{u v \in E(G)} 1\right)^{1 / 2}}^{\frac{1}{2}\left(\sqrt{\frac{\Delta+\delta}{2 \sqrt{\Delta \delta}}}+\sqrt{\frac{2 \sqrt{\Delta \delta}}{\Delta+\delta}}\right)}\right.}{} \\
& \geq \frac{2 \sqrt{2 \sqrt{\Delta \delta}(\Delta+\delta) m}}{(\sqrt{\Delta}+\sqrt{\delta})^{2}}\left(\sum_{u v \in E(G)} \frac{1}{\Delta^{2}} d_{u} d_{v}\right)^{1 / 2} \\
& =\frac{2 \sqrt{2 \sqrt{\Delta \delta}(\Delta+\delta) m M_{2}(G)}}{\Delta(\sqrt{\Delta}+\sqrt{\delta})^{2}} .
\end{aligned}
$$

Therefore,

$$
\frac{2 \sqrt{2 \sqrt{\Delta \delta}(\Delta+\delta) m M_{2}(G)}}{\Delta(\sqrt{\Delta}+\sqrt{\delta})^{2}} \leq G A_{1}(G)
$$


On the other hand,

$$
G A_{1}(G)=\sum_{u v \in E(G)} \frac{\sqrt{d_{u} d_{v}}}{\frac{1}{2}\left(d_{u}+d_{v}\right)}=\sum_{u v \in E(G)} \frac{d_{u} d_{v}}{\frac{1}{2}\left(d_{u}+d_{v}\right) \sqrt{d_{u} d_{v}}} \leq \sum_{u v \in E(G)} \frac{d_{u} d_{v}}{\delta \dot{\delta}} .
$$

Thus, we have

$$
G A_{1}(G) \leq \frac{M_{2}(G)}{\delta^{2}} .
$$

If the graph is regular, then $M_{2}(G)=\delta^{2} m$, the lower and upper bound are the same, and they are equal to $G A_{1}(G)=m$. If the equality holds in the lower bound, then $4\left(d_{u}+d_{v}\right)^{-2}=\Delta^{-2}$ for every $u v \in E(G)$; hence, $d_{u}=\Delta$ for every $u \in V(G)$ and the graph is regular. If the equality is attained in the upper bound, then $\frac{1}{2}\left(d_{u}+d_{v}\right)=$ $\delta$ for every $u v \in E(G)$ and we conclude $d_{u}=\delta$ for every $u \in V(G)$.

Theorem 1 improves the previous lower bound, as the following result shows.

Remark 1. For any $0<\delta \leq \Delta$ we have

$$
\frac{\sqrt{2 \sqrt{\Delta \delta}(\Delta+\delta)}}{\Delta(\sqrt{\Delta}+\sqrt{\delta})^{2}} \geq \frac{1}{\Delta+\delta} \sqrt{\frac{\delta}{\Delta}} .
$$

Proof. First of all, note that the polynomial $p(x)=2 x^{6}-x^{5}+2 x^{4}-6 x^{3}+2 x^{2}-$ $x+2$ verifies $p(x) \geq 0$ if $x \geq 1$, since $p(x)=(x-1)^{2}\left(2 x^{4}+3 x^{3}+6 x^{2}+3 x+2\right)$. Hence,

$$
\begin{aligned}
2 x^{6}+2 x^{4}+2 x^{2}+2 & \geq x\left(x^{4}+6 x^{2}+1\right), \quad \text { if } x \geq 1, \\
2\left(\frac{\Delta}{\delta}\right)^{3}+2\left(\frac{\Delta}{\delta}\right)^{2}+2 \frac{\Delta}{\delta}+2 & \geq \sqrt{\frac{\Delta}{\delta}}\left(\left(\frac{\Delta}{\delta}\right)^{2}+6 \frac{\Delta}{\delta}+1\right), \\
2 \Delta^{3}+2 \Delta^{2} \delta+2 \Delta \delta^{2}+2 \delta^{3} & \geq \Delta^{2} \sqrt{\Delta \delta}+6 \Delta \delta \sqrt{\Delta \delta}+\delta^{2} \sqrt{\Delta \delta}, \\
2(\Delta+\delta)^{3} & \geq \sqrt{\Delta \delta}(\sqrt{\Delta}+\sqrt{\delta})^{4},
\end{aligned}
$$

and this implies the inequality.

In [2] appears the inequality

$$
G A_{1}(G) \leq \frac{1}{2} M_{1}(G)
$$

In [16] appears the following result

$$
G A_{1}(G) \leq \frac{1}{2 \delta} M_{1}(G) .
$$

Our next result improves both inequalities and also gives a lower bound of $G A_{1}$ involving the first Zagreb index. 
Theorem 2. We have for any graph $G$

$$
\frac{2 \delta M_{1}(G)}{(\Delta+\delta)^{2}} \leq G A_{1}(G) \leq \sqrt{\frac{m M_{1}(G)}{2 \delta}} .
$$

Furthermore, the equality in each inequality is attained if and only if $G$ is regular.

Proof. By Lemma 1, taking $a=\delta$ and $b=\Delta$, we have

$$
\frac{2 \sqrt{\Delta \delta}}{\Delta+\delta} \leq \frac{2 \sqrt{d_{u} d_{v}}}{d_{u}+d_{v}} \leq 1
$$

Hence,

$$
1 \leq \frac{\left(d_{u}+d_{v}\right)^{2}}{4 d_{u} d_{v}} \leq \frac{(\Delta+\delta)^{2}}{4 \Delta \delta}, \quad \frac{d_{u}+d_{v}}{2 \sqrt{d_{u} d_{v}}} \leq \frac{(\Delta+\delta)^{2}}{4 \Delta \delta} \frac{2 \sqrt{d_{u} d_{v}}}{d_{u}+d_{v}} .
$$

Note that

$$
M_{1}(G)=\sum_{v \in V(G)} d_{v}^{2}=\sum_{u v \in E(G)}\left(d_{u}+d_{v}\right)
$$

Thus, we have

$$
\frac{M_{1}(G)}{2 \Delta}=\sum_{u v \in E(G)} \frac{d_{u}+d_{v}}{2 \Delta} \leq \sum_{u v \in E(G)} \frac{d_{u}+d_{v}}{2 \sqrt{d_{u} d_{v}}} \leq \frac{(\Delta+\delta)^{2}}{4 \Delta \delta} G A_{1}(G) .
$$

Therefore,

$$
\frac{2 \delta M_{1}(G)}{(\Delta+\delta)^{2}} \leq G A_{1}(G)
$$

Cauchy-Schwarz inequality and $\frac{1}{d_{u}+d_{v}} \leq \frac{1}{2 \delta}$ give

$$
\begin{aligned}
G A_{1}(G) & =\sum_{u v \in E(G)} \frac{2 \sqrt{d_{u} d_{v}}}{d_{u}+d_{v}} \leq \sum_{u v \in E(G)} \frac{\sqrt{d_{u}+d_{v}}}{\sqrt{d_{u}+d_{v}}} \\
& \leq\left(\sum_{u v \in E(G)}\left(d_{u}+d_{v}\right)\right)^{1 / 2}\left(\sum_{u v \in E(G)} \frac{1}{d_{u}+d_{v}}\right)^{1 / 2} \leq \sqrt{\frac{m M_{1}(G)}{2 \delta}} .
\end{aligned}
$$

If the graph is regular, then $M_{1}(G)=n \delta^{2}=2 m \delta$, the lower and upper bound are the same, and they are equal to $G A_{1}(G)=m$. If the equality holds in the lower bound, then $\sqrt{d_{u} d_{v}}=\Delta$ for every $u v \in E(G)$; hence, $d_{u}=\Delta$ for every $u \in V(G)$ and $G$ is regular. If the equality is attained in the upper bound, then $d_{u}+d_{v}=2 \delta$ for every $u v \in E(G)$ and we conclude $d_{u}=\delta$ for every $u \in V(G)$.

Note that Theorem 2 improves the upper bound $\frac{1}{2 \delta} M_{1}(G)$ : since $2 \delta m \leq M_{1}(G)$, we have

$$
\sqrt{\frac{m M_{1}(G)}{2 \delta}} \leq \frac{1}{2 \delta} M_{1}(G)
$$


Recall that the variable Zagreb index is defined as $Z_{\alpha}(G)=\sum_{u v \in E(G)}\left(d_{u} d_{v}\right)^{\alpha}$. Note that $Z_{-1 / 2}$ is the usual Randić index $R(G), Z_{1}$ is the second Zagreb index $M_{2}$, $Z_{-1}$ is the modified Zagreb index [12], etc.

Next, we obtain new inequalities relating the geometric-arithmetic index with the modified Zagreb index. We will need also the following lemmas.

Lemma 3. We have for any graph $G$ and every $u v \in E(G)$

$$
\delta \leq \frac{2 d_{u} d_{v}}{d_{u}+d_{v}} \leq \Delta,
$$

and the lower (or upper) bound is attained for every $u v \in E(G)$ if and only if $G$ is a regular graph.

Proof. We know that

$$
d_{u}\left(d_{v}-\delta\right)+d_{v}\left(d_{u}-\delta\right) \geq 0, \quad d_{u} d_{v}+d_{v} d_{u} \geq d_{u} \delta+d_{v} \delta .
$$

Therefore,

$$
\delta \leq \frac{2 d_{u} d_{v}}{d_{u}+d_{v}} .
$$

On the other hand, using the fact that the geometric mean is at most the arithmetic mean, we obtain

$$
\frac{2 d_{u} d_{v}}{d_{u}+d_{v}} \leq \frac{\left(d_{u}+d_{v}\right)^{2}}{2\left(d_{u}+d_{v}\right)}=\frac{d_{u}+d_{v}}{2} \leq \Delta .
$$

If $G$ is a regular graph, then it is clear that both inequalities are attained for every $u v \in E(G)$. Assume that the lower bound is attained for every $u v \in E(G)$. Then $d_{v}-\delta=d_{u}-\delta=0$ for every $u v \in E(G)$, and $d_{v}=\delta$ for every $v \in V(G)$. Hence, $G$ is a regular graph. If the upper bound is attained for every $u v \in E(G)$, then $4 d_{u} d_{v}=\left(d_{u}+d_{v}\right)^{2}$ for every $u v \in E(G), d_{u}=d_{v}$ for every $u v \in E(G)$, and we conclude that $G$ is regular since it is connected.

In order to prove our next result we need the following particular case of Jensen's inequality.

Lemma 4. If $f$ is a convex function in $\mathbb{R}_{+}$and $x_{1}, \ldots, x_{m}>0$, then

$$
f\left(\frac{x_{1}+\cdots+x_{m}}{m}\right) \leq \frac{1}{m}\left(f\left(x_{1}\right)+\cdots+f\left(x_{m}\right)\right) .
$$

Theorem 3. We have for any graph $G$

$$
\sqrt{\frac{m^{3}}{\Delta^{2} Z_{-1}(G)}} \leq G A_{1}(G) \leq \Delta^{2} Z_{-1}(G) .
$$

Furthermore, the equality in each inequality is attained if and only if $G$ is regular. 
Proof. Using Lemma 3, we have

$$
\begin{gathered}
\delta \leq \frac{2 d_{u} d_{v}}{d_{u}+d_{v}} \leq \Delta, \\
\frac{\delta}{d_{u} d_{v}} \leq \frac{2}{d_{u}+d_{v}} \leq \frac{\Delta}{d_{u} d_{v}} .
\end{gathered}
$$

Moreover,

$$
\sum_{u v \in E(G)} \frac{2 \sqrt{d_{u} d_{v}}}{d_{u}+d_{v}} \leq \Delta \sum_{u v \in E(G)} \frac{2}{d_{u}+d_{v}} \leq \Delta \sum_{u v \in E(G)} \frac{\Delta}{d_{u} d_{v}}=\Delta^{2} Z_{-1}(G)
$$

Since $f(x)=1 / x^{2}$ is a convex function in $\mathbb{R}_{+}$, Lemma 4 gives

$$
\begin{aligned}
\frac{m^{2}}{\left(\sum_{u v \in E(G)} \frac{2 \sqrt{d_{u} d_{v}}}{d_{u}+d_{v}}\right)^{2}} & \leq \frac{1}{m} \sum_{u v \in E(G)} \frac{\left(d_{u}+d_{v}\right)^{2}}{4 d_{u} d_{v}} \leq \frac{\Delta^{2}}{m} \sum_{u v \in E(G)} \frac{1}{d_{u} d_{v}}, \\
\frac{m^{2}}{\left(G A_{1}(G)\right)^{2}} & \leq \frac{\Delta^{2}}{m} Z_{-1}(G) .
\end{aligned}
$$

If the graph is regular, then $Z_{-1}(G)=m \Delta^{-2}$, the lower and upper bound are the same, and they are equal to $G A_{1}(G)=m$. If the equality holds in the upper bound, then $\sqrt{d_{u} d_{v}}=\Delta$ for every $u v \in E(G)$; hence, $d_{u}=\Delta$ for every $u \in V(G)$ and the graph is regular. If the equality is attained in the lower bound, then $\left(d_{u}+d_{v}\right)^{2}=4 \Delta^{2}$ for every $u v \in E(G)$ and we conclude $d_{u}=\Delta$ for every $u \in V(G)$.

The following result gives a lower and upper bounds for $G A_{1}$ involving the Zagreb indices $M_{2}(G)$ and $Z_{-1}(G)$.

Theorem 4. We have for any graph $G$

$$
\frac{2 \delta^{2} \sqrt{M_{2}(G) Z_{-1}(G)}}{\Delta^{2}+\delta^{2}} \leq G A_{1}(G) \leq \sqrt{M_{2}(G) Z_{-1}(G)},
$$

and the equality in each inequality is attained if and only if $G$ is a regular graph.

Proof. Lemma 3 gives

$$
\delta \leq \frac{2 d_{u} d_{v}}{d_{u}+d_{v}} \leq \Delta
$$

It is easy to see that

$$
\frac{1}{\Delta} \leq \frac{1}{\sqrt{d_{u} d_{v}}} \leq \frac{1}{\delta}
$$


Thus Lemma 2 gives

$$
\begin{aligned}
& G A_{1}(G)=\sum_{u v \in E(G)} \frac{\sqrt{d_{u} d_{v}}}{\frac{1}{2}\left(d_{u}+d_{v}\right)}=\sum_{u v \in E(G)} \frac{2 d_{u} d_{v}}{d_{u}+d_{v}} \frac{1}{\sqrt{d_{u} d_{v}}} \\
& \geq \frac{\left(\sum_{u v \in E(G)} \frac{4\left(d_{u} d_{v}\right)^{2}}{\left(d_{u}+d_{v}\right)^{2}}\right)^{1 / 2}\left(\sum_{u v \in E(G)} \frac{1}{d_{u} d_{v}}\right)^{1 / 2}}{\frac{1}{2}\left(\frac{\Delta}{\delta}+\frac{\delta}{\Delta}\right)} \\
& \geq \frac{2 \Delta \delta \sqrt{Z_{-1}(G)}}{\Delta^{2}+\delta^{2}}\left(\sum_{u v \in E(G)} \frac{4\left(d_{u} d_{v}\right)^{2}}{\left(d_{u}+d_{v}\right)^{2}}\right)^{1 / 2} \\
& \geq \frac{2 \Delta \delta \sqrt{Z_{-1}(G)}}{\Delta^{2}+\delta^{2}} \frac{2 \delta}{2 \Delta}\left(\sum_{u v \in E(G)} d_{u} d_{v}\right)^{1 / 2} \\
& =\frac{2 \delta^{2} \sqrt{M_{2}(G) Z_{-1}(G)}}{\Delta^{2}+\delta^{2}} .
\end{aligned}
$$

Since

$$
4 d_{u} d_{v} \leq\left(d_{u}+d_{v}\right)^{2}
$$

Cauchy-Schwarz inequality gives

$$
\begin{aligned}
G A_{1}(G) & =\sum_{u v \in E(G)} \frac{2 \sqrt{d_{u} d_{v}}}{d_{u}+d_{v}} \leq\left(\sum_{u v \in E(G)} d_{u} d_{v}\right)^{1 / 2}\left(\sum_{u v \in E(G)} \frac{4}{\left(d_{u}+d_{v}\right)^{2}}\right)^{1 / 2} \\
& \leq\left(\sum_{u v \in E(G)} d_{u} d_{v}\right)^{1 / 2}\left(\sum_{u v \in E(G)} \frac{1}{d_{u} d_{v}}\right)^{1 / 2}=\sqrt{M_{2}(G) Z_{-1}(G)} .
\end{aligned}
$$

If the graph is regular, then the lower and upper bound are the same, and they are equal to $G A_{1}(G)$. If the equality holds in the lower bound, then $d_{u} d_{v}=\delta^{2}$ for every $u v \in E(G)$; hence, $d_{u}=\delta$ for every $u \in V(G)$ and the graph is regular. If the equality is attained in the upper bound, then $4 d_{u} d_{v}=\left(d_{u}+d_{v}\right)^{2}$ for every $u v \in E(G), d_{u}=$ $d_{v}$ for every $u v \in E(G)$, and we conclude that $G$ is regular since it is connected.

Next, we obtain inequalities relating the geometric-arithmetic index with the Zagreb indices $M_{1}(G), M_{2}(G)$ and $Z_{-1}(G)$. Recall that a $(\Delta, \delta)$-biregular graph is a bipartite graph for which any vertex in one side of the given bipartition has degree $\Delta$ and any vertex in the other side of the bipartition has degree $\delta$.

Theorem 5. For any graph $G, \frac{\Delta \delta \sqrt{\left(n M_{1}(G)+4 M_{2}(G)-4 m^{2}\right) Z_{-1}(G)}}{\Delta^{2}+\delta^{2}} \leq G A_{1}(G) \leq$ $\frac{\sqrt{\left(n M_{1}(G)+4 M_{2}(G)-4 m^{2}\right) Z_{-1}(G)}}{2}$. The equality in the lower bound is attained if and only if $G$ is either regular or $(\Delta, \delta)$-biregular; the equality in the upper bound is attained if and only if $G$ is regular. 
Proof. We have by Lemma 1

$$
\frac{4 \Delta \delta}{(\Delta+\delta)^{2}} \leq \frac{4 d_{u} d_{v}}{\left(d_{u}+d_{v}\right)^{2}} \leq 1
$$

Hence,

$$
\frac{2 \sqrt{d_{u} d_{v}}}{d_{u}+d_{v}} \leq \frac{d_{u}+d_{v}}{2 \sqrt{d_{u} d_{v}}}
$$

and

$$
\frac{2 \sqrt{d_{u} d_{v}}}{d_{u}+d_{v}} \geq \frac{4 \Delta \delta}{(\Delta+\delta)^{2}} \frac{d_{u}+d_{v}}{2 \sqrt{d_{u} d_{v}}}
$$

We have by (2.1)

$$
G A_{1}(G)=\sum_{u v \in E(G)} \frac{2 \sqrt{d_{u} d_{v}}}{d_{u}+d_{v}} \leq \sum_{u v \in E(G)} \frac{d_{u}+d_{v}}{2 \sqrt{d_{u} d_{v}}} .
$$

Moreover,

$$
\begin{aligned}
\sum_{u v \in E(G)}\left(d_{u}+d_{v}\right)^{2} & =\sum_{u v \in E(G)} d_{u}^{2}+2 \sum_{u v \in E(G)} d_{u} d_{v}+\sum_{u v \in E(G)} d_{v}^{2} \\
& =\sum_{u v \in E(G)}\left(d_{u}^{2}+d_{v}^{2}\right)+2 \sum_{u v \in E(G)} d_{u} d_{v} \\
& =\sum_{u v \in E(G)}\left(d_{u}^{2}+d_{v}^{2}\right)+2 M_{2}(G) .
\end{aligned}
$$

Since

$$
\sum_{u v \in E(G)}\left(d_{u}^{2}+d_{v}^{2}\right)=\sum_{v \in V(G)} d_{v} \cdot d_{v}^{2}=\sum_{v \in V(G)} d_{v}^{3}
$$

we have

$$
\sum_{u v \in E(G)}\left(d_{u}+d_{v}\right)^{2}=\sum_{v \in V(G)} d_{v}^{3}+2 M_{2}(G) .
$$

Using Cauchy-Schwarz inequality and the formula

$$
\sum_{v \in V(G)} d_{v}^{3}=n M_{1}(G)+2 M_{2}(G)-4 m^{2}
$$


(see, e.g., [21]), we obtain

$$
\begin{aligned}
\sum_{u v \in E(G)} \frac{d_{u}+d_{v}}{2 \sqrt{d_{u} d_{v}}} & \leq\left(\sum_{u v \in E(G)} \frac{\left(d_{u}+d_{v}\right)^{2}}{4}\right)^{1 / 2}\left(\sum_{u v \in E(G)} \frac{1}{d_{u} d_{v}}\right)^{1 / 2} \\
& =\frac{1}{2}\left(\sum_{v \in V(G)} d_{v}^{3}+2 M_{2}(G)\right)^{1 / 2} \sqrt{Z_{-1}(G)} \\
& =\frac{\sqrt{\left(n M_{1}(G)+4 M_{2}(G)-4 m^{2}\right) Z_{-1}(G)}}{2}
\end{aligned}
$$

Let us prove the lower bound. We have by (2.2)

$$
G A_{1}(G) \geq \frac{4 \delta \Delta}{(\delta+\Delta)^{2}} \sum_{u v \in E(G)} \frac{d_{u}+d_{v}}{2 \sqrt{d_{u} d_{v}}} .
$$

Since

$$
\delta \leq \frac{d_{u}+d_{v}}{2} \leq \Delta, \quad \frac{1}{\Delta} \leq \frac{1}{\sqrt{d_{u} d_{v}}} \leq \frac{1}{\delta},
$$

Lemma 2, (2.3) and (2.4) give

$$
\begin{aligned}
\sum_{u v \in E(G)} \frac{d_{u}+d_{v}}{2 \sqrt{d_{u} d_{v}}} & \geq \frac{\left(\sum_{u v \in E(G)} \frac{\left(d_{u}+d_{v}\right)^{2}}{4}\right)^{1 / 2}\left(\sum_{u v \in E(G)} \frac{1}{d_{u} d_{v}}\right)^{1 / 2}}{\frac{1}{2}\left(\frac{\Delta}{\delta}+\frac{\delta}{\Delta}\right)} \\
& \geq \frac{\Delta \delta \sqrt{\left(\sum_{v \in V(G)} d_{v}^{3}+2 M_{2}(G)\right) Z_{-1}(G)}}{\Delta^{2}+\delta^{2}} \\
& =\frac{\Delta \delta \sqrt{\left(n M_{1}(G)+4 M_{2}(G)-4 m^{2}\right) Z_{-1}(G)}}{\Delta^{2}+\delta^{2}} .
\end{aligned}
$$

If the graph is regular, then the lower and upper bound are the same, and they are equal to $G A_{1}(G)$.

If the equality in the lower bound is attained, then (2.2) holds for every $u v \in E(G)$; by Lemma 1 we have either $d_{u}=\Delta$ and $d_{v}=\delta$, or viceversa, for each $u v \in E(G)$. This happens if and only if $G$ is a regular graph if $\Delta=\delta$ or a $(\Delta, \delta)$-biregular graph otherwise.

If the equality in the upper bound is attained, then (2.1) holds for every $u v \in E(G)$; by Lemma 1 we have $d_{u}=d_{v}$ for each $u v \in E(G)$, and $G$ is a regular graph.

In [16] we find the bound

$$
G A_{1}(G) \leq \Delta R(G),
$$

where $R(G)$ is the Randić index. We recall that $R(G)$ is the best know topological index. 
The following result provides lower and upper bounds of $G A_{1}$ involving the Randić index. Furthermore, it improves (2.5).

Theorem 6. We have for any graph $G$

$$
\delta R(G) \leq G A_{1}(G) \leq \sqrt{\Delta m R(G)},
$$

and the equality in each inequality holds if and only if $G$ is regular.

Proof. Since $f(x)=x^{2}$ is a convex function in $\mathbb{R}_{+}$, Lemma 4 gives

$$
\frac{1}{m^{2}} G A_{1}(G)^{2}=\frac{1}{m^{2}}\left(\sum_{u v \in E(G)} \frac{2 \sqrt{d_{u} d_{v}}}{d_{u}+d_{v}}\right)^{2} \leq \frac{1}{m} \sum_{u v \in E(G)} \frac{4 d_{u} d_{v}}{\left(d_{u}+d_{v}\right)^{2}} .
$$

Lemma 3 gives

$$
\begin{aligned}
\frac{2 d_{u} d_{v}}{d_{u}+d_{v}} & \leq \Delta, \\
\frac{2 d_{u} d_{v}\left(2 \sqrt{d_{u} d_{v}}\right)}{\left(d_{u}+d_{v}\right)^{2}} & \leq \frac{2 d_{u} d_{v}\left(d_{u}+d_{v}\right)}{\left(d_{u}+d_{v}\right)^{2}}=\frac{2 d_{u} d_{v}}{d_{u}+d_{v}} \leq \Delta, \\
\frac{4 d_{u} d_{v}}{\left(d_{u}+d_{v}\right)^{2}} & \leq \frac{\Delta}{\sqrt{d_{u} d_{v}}}, \\
\frac{1}{m^{2}} G A_{1}(G)^{2} & \leq \frac{1}{m} \sum_{u v \in E(G)} \frac{4 d_{u} d_{v}}{\left(d_{u}+d_{v}\right)^{2}} \leq \frac{\Delta}{m} \sum_{u v \in E(G)} \frac{1}{\sqrt{d_{u} d_{v}}} .
\end{aligned}
$$

Hence,

$$
G A_{1}(G) \leq \sqrt{\Delta m R(G)} .
$$

In order to prove the lower bound, notice that Lemma 3 gives

$$
\delta \leq \frac{2 d_{u} d_{v}}{d_{u}+d_{v}}=\frac{2 \sqrt{d_{u} d_{v}} \sqrt{d_{u} d_{v}}}{d_{u}+d_{v}} .
$$

Hence,

$$
\begin{aligned}
\sum_{u v \in E(G)} \frac{\delta}{\sqrt{d_{u} d_{v}}} & \leq \sum_{u v \in E(G)} \frac{\sqrt{d_{u} d_{v}}}{\frac{1}{2}\left(d_{u}+d_{v}\right)}, \\
\delta R(G) & \leq G A_{1}(G) .
\end{aligned}
$$

If the graph is regular, then $R(G)=\frac{m}{\delta}$, the lower and upper bound are the same, and they are equal to $G A_{1}(G)=m$.

If the equality in the lower bound is attained, then

$$
\delta=\frac{2 d_{u} d_{v}}{d_{u}+d_{v}}
$$

for every $u v \in E(G)$. By Lemma 3, the graph $G$ is regular. 
If the equality in the upper bound is attained, then

$$
\Delta=\frac{2 d_{u} d_{v}}{d_{u}+d_{v}}
$$

for every $u v \in E(G)$, and $G$ is regular by Lemma 3 .

Note that Theorem 6 improves the upper bound $G A_{1}(G) \leq \Delta R(G)$ : since $\frac{m}{\Delta} \leq$ $R(G)$, we have

$$
\sqrt{\Delta m R(G)} \leq \Delta R(G)
$$

\section{ACKNOWLEDGEMENT}

We would like to thank the referees for their careful reading of the manuscript and several useful comments which have helped us to improve the presentation of the paper. Moreover, we are grateful for discussions with J. M. Rodríguez on some early version of this paper. The author was partially supported by two grants from Ministerio de Economía y Competitividad (MTM 2013-46374), Spain and CONACYT (2015), Mexico.

\section{REFERENCES}

[1] V. Andova, N. Cohen, and R. Škrekovski, "A note on zagreb indices inequality for trees and unicyclic graphs," ARS Math. Contemp., vol. 5, pp. 73-76, 2012.

[2] K. C. Das and I. Gutman, "Survey on geometric-arithmetic indices of graphs," MATCH Commun. Math. Comput. Chem., vol. 65, pp. 595-644, 2011.

[3] K. C. Das, I. Gutman, and B. Furtula, "On first geometric-arithmetic index of graphs,” Discrete Appl. Math., vol. 159, pp. 2030-2037, 2011, doi: 10.1016/j.dam.2011.06.020.

[4] K. C. Das, D. W. Lee, and A. Graovac, "Some properties of the zagreb eccentricity indices," ARS Math. Contemp., vol. 6, pp. 117-125, 2013.

[5] T. Dehghan, H. Hua, A. R. Ashrafi, and N. Hababi, "Remark on a conjecture about randić index and graphs radius," Miskolc Math. Notes, vol. 14, no. 3, pp. 845-850, 2013.

[6] B. Furtula, I. Gutman, and S. Ediz, "On difference of zagreb indices," Discrete Appl. Math., vol. 178, pp. 83-88, 2014, doi: 10.1016/j.dam.2014.06.011.

[7] I. Gutman, "N. trinajstić, graph theory and molecular orbitals. total $\pi$-electron energy of alternant hydrocarbons," Chem. Phys. Lett., vol. 17, pp. 535-538, 1972, doi: 10.1016/0009-2614(72)850991 .

[8] G. H. Hardy, J. E. Littlewood, and G. Polya, Inequalities, 2nd ed. Cambridge: Cambridge University Press, 1952.

[9] X. Li and I. Gutman, Mathematical Aspects of Randić Type Molecular Structure Descriptors, ser. Mathematical Chemistry Monographs. Kragujevac: Univ. Kragujevac, 2006.

[10] X. Li and Y. Shi, "A survey on the randić index," MATCH Commun. Math. Comput. Chem., vol. 59, pp. 127-156, 2008.

[11] A. Miličević and S. Nikolić, "On variable zagreb indices," Croat. Chem. Acta, vol. 77, pp. 97-101, 2004.

[12] S. Nikolić, G. Kovačević, A. Miličević, and N. Trinajstić, "The zagreb indices 30 years after," Croat. Chem. Acta, vol. 76, pp. 113-124, 2003.

[13] J. Rada and R. Cruz, "Vertex-degree-based topological indices over graphs," MATCH Commun. Math. Comput. Chem., vol. 72, pp. 603-616, 2014. 
[14] M. Randić, "On characterization of molecular branching," J. Am. Chem. Soc., vol. 97, pp. 66096615, 1975.

[15] J. A. Rodríguez and J. M. Sigarreta, "On the randić index and condicional parameters of a graph," MATCH Commun. Math. Comput. Chem., vol. 54, pp. 403-416, 2005.

[16] J. M. Rodríguez and J. M. Sigarreta, "On the geometric-arithmetic index," MATCH Commun. Math. Comput. Chem., vol. 74, pp. 103-120, 2015.

[17] J. M. Rodríguez and J. M. Sigarreta, "Spectral study of the geometric-arithmetic index," MATCH Commun. Math. Comput. Chem., vol. 74, pp. 121-135, 2015.

[18] D. Vukičević and B. Furtula, "Topological index based on the ratios of geometrical and arithmetical means of end-vertex degrees of edges," J. Math. Chem., vol. 46, pp. 1369-1376, 2009, doi: 10.1007/s10910-009-9520-x.

[19] H. Wiener, "Structural determination of paraffin boiling points," J. Am. Chem. Soc., vol. 69, pp. 17-20, 1947, doi: 10.1021/ja01193a005.

[20] S. H. Zadeh, A. Hamzeh, and A. R. Ashrafi, "Extremal properties of zagred coindices and degree distance of graphs," Miskolc Math. Notes, vol. 11, no. 2, pp. 129-137, 2010.

[21] B. Zhou, "Remarks on zagreb indices," MATCH Commun. Math. Comput. Chem., vol. 57, pp. 591-596, 2007.

[22] B. Zhou, I. Gutman, B. Furtula, and Z. Du, "On two types of geometric-arithmetic index," Chem. Phys. Lett., vol. 482, pp. 153-155, 2009, doi: 10.1016/j.cplett.2009.09.102.

Author's address

José M. Sigarreta

Universidad Autónoma de Guerrero, Facultad de Matemáticas, Carlos E. Adame No.54 Col. Garita, 39650 Acapulco, México

E-mail address: josemariasigarretaalmira@hotmail.com 